\title{
DIRECT DETECTION OF BORRELIA BURGDORFERI SPIROCHETES IN PATIENTS WITH EARLY DISSEMINATED LYME BORRELIOSIS
}

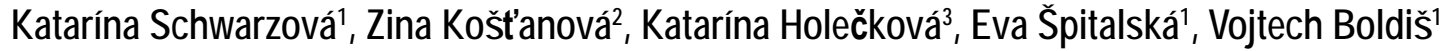 \\ ${ }^{1}$ Institute of Virology, Slovak Academy of Sciences, Bratislava \\ ${ }^{2}$ Regional Office of Public Health, Žiar nad Hronom \\ ${ }^{3}$ Slovak Health University, Bratislava, Slovak Republic
}

\begin{abstract}
SUMMARY
The detection of spirochetes in 15 patients with clinically documented early disseminated LB has been analysed when using cultivation method of the plasma or the cerebrospinal fluid, electron microscopy, commercial Western blot and detecting the DNA of the pathogen in vitro cultures by PCR-RFLP. Spirochetes were isolated in eight blood and one cerebrospinal fluid culture samples. In seven cases (47\%), previous serodiagnostic laboratory tests were negative. Borrelial DNA was detected by PCR in 67\% patients (9 blood samples and 1 CSF sample). Using Msel restriction fragments of PCR products of the amplified rrf-rrl region, we identified Borrelia garinii (80\%), one B. afzelii isolate and one B. burgdorferi s.s.
\end{abstract}

Key words: Borrelia burgdorferi, direct detection, cultivation, electron microscopy, polymerase chain reaction (PCR), Restriction fragment length polymorphism (RFLP) analysis

Address for correspondence: K. Schwarzová, Institute of Virology, SlovakAcademy of Sciences, Dúbravská cesta 9, 84505 Bratislava, Slovakia. E-mail: virukats@savba.sk

\section{INTRODUCTION}

Due to the global changes (climate, economy, social) in the last decade there is a spread of the new emerging infectious diseases of various etiology. Reported cases of zoonotic diseases are growing also due to the improved diagnostic procedures. Lyme borreliosis (LB) is the example of such trend. It is not a new epidemic, but the better diagnosed and recognised disease. This multi-organ infection is caused by spirochetes of the Borrelia burgdorferi sensu lato group with species $B$. burgdorferi sensu stricto ( $B$. burgdorferi s.s.), B. garinii and B. afzelii, which are transmitted by ticks of the species Ixodes. B. burgdorferi s.l. has been isolated from different clinical specimens: CSF, blood, synovial fluid, during early as well as chronic stages of LB $(1,2,3)$. Serological tests for detection of borrelial antibodies are frequently used in laboratory diagnosis of Lyme borreliosis. Unfortunately these tests are not standardized and the results obtained with different assays may not be sufficiently conclusive $(4,5)$.

Objective: The study is focused on the problem of laboratory diagnosis of Lyme disease. The aim of this analysis was the detection of B.b. s.l. or its DNA presence in 15 samples from patients with previous negative serological examination of IgM or IgG specific antibodies in assays using commercial method (ELISA).

\section{MATERIAL AND METHODS}

\section{Collection of Patient Samples}

The study included 15 patients diagnosed with early disseminated LB during 2006-2007. The data about patients concerning age, sex, presence of Borrelia burgdorferi s. I. IgG and IgM antibodies (tested by ELISA kit), tick-bite and disease symptoms were obtained in cooperation with Department of Infectious and Geographical Medicine, Bratislava and medical workers in districts of Žiar n/Hronom. The blood samples from 15 patients was analysed. In four cases also CSF was examined. Ten of those (67\%) were children with average age of 9 (ranging from 7-15 years) and 5 (33\%) were adult. Within the group of five adult patients, 3 were women with average age of 47 (ranging from 43 to 51 years) and 2 were man with age of 55 and 60 years. Five patients suffered from neuroborreliosis (33\%), 8 patients (53\%) had erythema migrans (EM) with neurological complications and in 2 patients (13\%) with sclerosis multiplex and arthritis LB was suspected. Samples of human blood and cerebrospinal fluid (CSF) were obtained prior to antibiotic therapy initiation.

Abbreviations used: PCR - Polymerase Chain Reaction, RFLP- Restriction Fragment Length Polymorphism, WB - Westernblot, EM - Erythema Migrans, CSF - Cerebrospinal Fluid, B.b.s.l. - Borrelia burgdorferi sensu lato, LB - Lyme borreliosis. 


\section{Cultivation of Borreliae from Blood and CSF}

Blood samples from all 15 patients in a volume of $5 \mathrm{ml}$ and cerebrospinal fluid samples $(3 \mathrm{ml}$ ) from four patients with neuroborreliosis involved in this study were centrifuged at 8000 rpm for $5 \mathrm{~min}$. The inoculum was transferred into one to three tubes containing BSK-H medium (Sigma) under microaerophilic conditions at $34{ }^{\circ} \mathrm{C}$ as described previously (6) and checked for the presence of borreliae weekly. Stock solutions were stored at $-70{ }^{\circ} \mathrm{C}$ until used.

Serology: Samples were tested with the commercial Borrelia garinii Westernblot IgG and IgM kits (Anti-Borrelia garinii-Western Blot IgM and IgG, Euroimmun, Germany), with electrophoretically separated antigens from the manufacturer. Interpretation of the results obtained with the use of commercial kits followed instruction of the manufacturer.

Bacterial strains: Isolates of $B$. burgdorferi s.l. complex served as control strains: strain MIL (B. burgdorferi s.s), VS461 (B. afzelii) and K10 (B. garinii). Isolate K10 was obtained from prof. Kmety (7). Strains VS461 and MIL were kindly provided by D. Postic, Pasteur Institute, Paris, France.

Electron microscopy: Isolates ( $>100$ microorganisms per $\mu \mathrm{l}$ of culture) were examined by electron microscopy according to Hovind-Hougen (8) as already described (6).

DNA extraction: DNA from primary culture tubes (a total of $5 \mathrm{ml}$ of the content) was extracted using the QIAamp DNA Blood Mini kit (Qiagen, Germany) following manufacturer's recommended protocol. Extracted DNA was stored at $-20^{\circ} \mathrm{C}$.

\section{PCR and Restriction Fragment Length Polymor- phism (RFLP) Analysis}

A total of $1 \mu \mathrm{l}$ of template DNA was added to a PCR master mixture containing $10 \mu \mathrm{l}$ of 2x PCR Master Mix (Fermentas),
10 pmol of each $(1 \mu \mathrm{l})$ specific primers and $7 \mu \mathrm{l}$ of deionised water. PCR amplification of the 5S-23S intergenic spacer region of Borrelia burgdorferi s.l. was performed as described by Postic et al. (9). The PCR products were digested with MseI according to the manufacturer's protocol (Fermentas) and identified in $2 \%$ agarose gel, stained with ethidium bromide.

\section{RESULTS}

A total of 15 clinical specimens were used in this study (Table 1). Spirochetes were detected from ten culture samples (9 cultivated blood and one cerebrospinal fluid samples), in 7 cases with previous negative serodiagnostic laboratory test results. In one case (patient No. 4), borreliae were isolated from the cerebrospinal fluid (CSF) sample (B. afzelii). Spirochetes (B. garinii) were detected from a patient suffering from sclerosis multiplex for several years (patient No. 11). This patient was bitten by the tick three weeks before this analysis. The direct detection of borrelial DNA in her blood was the reason for the antibiotic treatment.

Spirochetal cells were detected in primary culture tubes after an incubation period of four weeks. The typical morphology of spirochetal cells was observed in electron microscopy. Several blebs and chains of granules could be observed at the outer envelope.

The samples were analyzed by PCR with primers specific to Borrelia pathogenic species. Based on 5S-23S rRNA intergenic spacer RFLP analysis using endonuclease MseI and reactivity with specific primers, cultures were in 8 cases identified as Borrelia garinii (80\%), one isolate $B$. afzelii and one B. burgdorferi s.s. (Fig. 1). In one case (patient No. 11) the culture was positive only by the PCR analysis.

Table 1. Review of patients with early disseminated stage of Lyme borreliosis screened by four distinct detection methods

\begin{tabular}{|c|c|c|c|c|c|c|c|c|}
\hline \multirow{2}{*}{ No. } & \multicolumn{2}{|c|}{ Patients } & \multicolumn{2}{|c|}{ ELISA } & \multicolumn{2}{|c|}{ WB } & \multirow{2}{*}{ Culture } & \multirow{2}{*}{ PCR } \\
\hline & Age & Sex & $\lg M$ & $\lg G$ & $\lg M$ & $\lg G$ & & \\
\hline 1 & 7 & $\mathrm{~F}$ & - & - & - & eqv & - & - \\
\hline 2 & 8 & $\mathrm{~F}$ & + & + & + & + & + & B.g. \\
\hline 3 & 8 & $\mathrm{M}$ & + & + & + & + & + & B.g. \\
\hline 4 & 9 & $\mathrm{~F}$ & - & - & - & - & + & B.a. \\
\hline 5 & 9 & $M$ & - & - & - & - & - & - \\
\hline 6 & 10 & $\mathrm{M}$ & eqv & - & - & - & + & B.g. \\
\hline 7 & 11 & $\mathrm{~F}$ & - & - & - & - & + & B.g. \\
\hline 8 & 11 & M & - & - & - & - & - & - \\
\hline 9 & 14 & $\mathrm{~F}$ & - & - & + & + & + & B.g. \\
\hline 10 & 15 & $\mathrm{~F}$ & - & - & - & - & + & B.g. \\
\hline 11 & 43 & $\mathrm{~F}$ & eqv & - & - & - & - & B.g. \\
\hline 12 & 47 & $\mathrm{~F}$ & - & - & - & - & - & - \\
\hline 13 & 51 & $\mathrm{~F}$ & - & - & - & - & + & B.b.s.s \\
\hline 14 & 55 & $\mathrm{M}$ & - & - & - & - & + & B.g. \\
\hline 15 & 60 & $M$ & eqv & - & - & - & - & - \\
\hline
\end{tabular}

Notes:

$(+)$ - anti-Borrelia IgM or IgG are present, positive; (-) - anti-Borrelia IgM or IgG are not present, negative; eqv - equivocal; F - female; M - male; B.a - Borrelia afzelii; B.g - Borrelia garinii; B.b.s.s. - Borrelia burgdorferi s.s. 


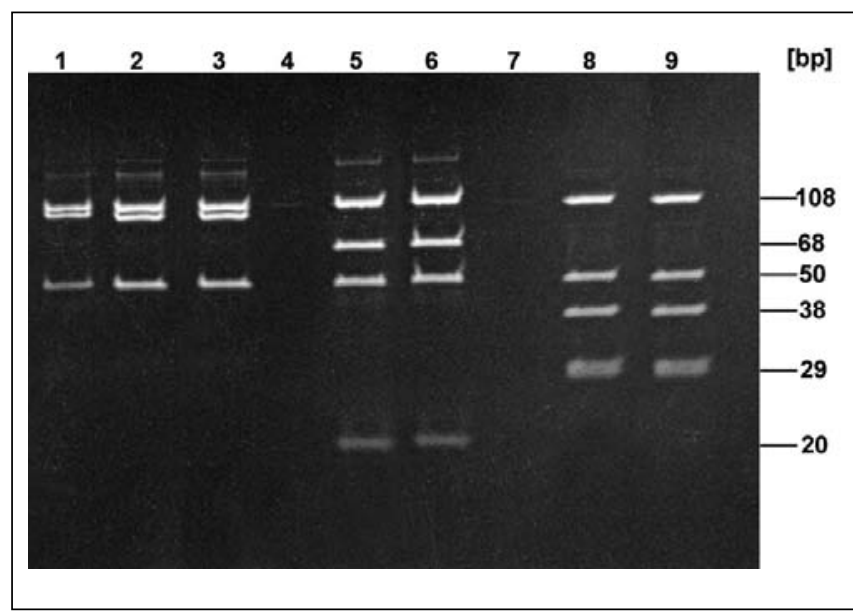

Fig. 1. Representative RFLP fragments of B. burgdorferi s.I. patients isolates:

1 - K10 (control strain, B. garinii), 2 - PK1 (B. garinii, patient isolate), 3 - MJ4 (B. garinii, patient isolate), 5 - VS461 (control strain, B. afzelii), 6 - LQ1 (B. afzelii, patient isolate), 8 - MIL (control strain, B. burgdorferi s.s.), 9 - isolate PR3 (B. burgdorferi s.s., patient isolate).

\section{DISCUSSION}

The presence of Borrelia burgdorferi s.l. (B. garinii, B. afzelii and $B$. burgdorferi s.s.) was demonstrated by culture in nine blood samples and one cerebrospinal fluid sample collected from 15 patients (67\%). Seven samples (47\%) were negative in previous commercial serodiagnostic laboratory tests. In one case, spirochetes were isolated from the CSF culture specimen (B. afzelii).

We observed several blebs and granules at the outer envelope in electron microscopy. Such degenerative products of Borrelia should be investigated in view of their possible contribution to the pathogenesis of LB (10).

LB is on the top of the list of statistically registered tick born diseases in Slovakia. From the database of the information system for reported communicable diseases (EPIS) it is apparent that the LB average morbidity was $12.81 / 100,000$ inhabitants in the last 10 years (1997-2007) (Fig. 2). Comparing the 5-years average an increase in the number of cases by $20 \%$ was noted (11).

Sensitivity of serodiagnostic tests varied with stage of the disease. The raise in the morbidity of this disease has been observed in the recent years, particularly in the group of advanced, disseminated stages of the disease. From EPIS it comes out that by the reported cases of LB with positive tick history and by the presence of the clinical signs, the serology with commercial tests was negative in $10 \%$ in patients with the diagnosis A69.2 (EM). In cases with the diagnosis G63.0 (early disseminated infection) the serology was negative in $13 \%$ and in those diagnosed with M01.2 (late disseminated stage of LB) in 6\% (11).

One of the reasons contributing to this situation seems to be the problem with the diagnosis of atypical clinical cases in early stages of LB development and insufficient differential diagnosis in disseminated forms of LB. Until now there is no sensitive and specific laboratory serodiagnostic test for this disease.

Applying local whole cell antigens usually seems to be more specific in comparison with commercial tests $(5,12)$. The data obtained by these methods must be interpreted in the context of the clinical finding and epidemiological history. Application of the optimal borrelia antigens is one of the factors of successful development of such tests.

In the field of LB diagnosis it is recommended to apply also PCR, or other detection methods and/or cultivation as a complementary method to the serology. PCR analysis appears to be reliable for the detection of the pathogen in the patient's samples. It also enables to differentiate genospecies $(13,14)$. However it also indicates possible co-infection by several genospecies in the same sample.

\section{CONCLUSIONS}

Absence of immune response does not mean the absence of borrelial infection, on the contrary, presence of an immune response does not indicate active infection. Direct detection (in vitro cultivation, PCR) of B. burgdorferi s.l. as well as other tick borne pathogens in the body liquids of the patients and animals is very important for diagnosis of the disease. It helps to confirm the infection when the results from immunological testing and

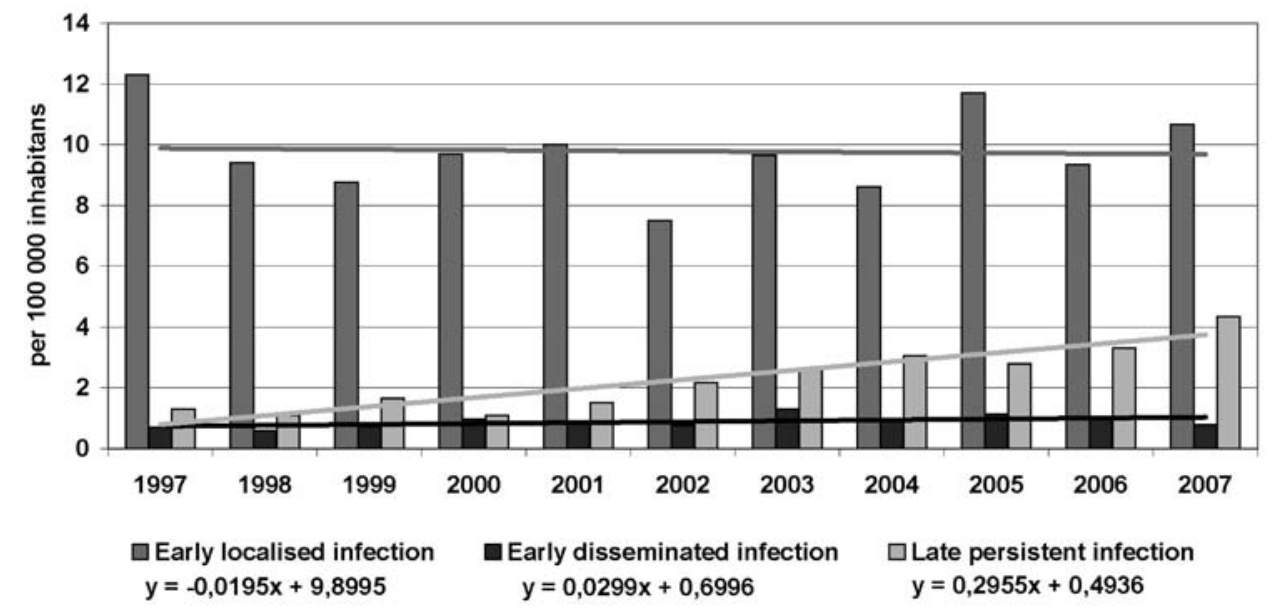

Fig. 2. Trends of morbidity of LB single stages in Slovakia from 1997-2007. 
other reactions of the macroorganism do not definitely confirm the disease. It helps also to indicate a potential presence of other pathogens in the patient.

The immunogenic role of outer surface proteins from local isolates of B. burgdorferi s.l. obtained from seronegative Lyme disease patients deserves further investigation. It may contribute to understanding of the pathogenesis and immunity in this complex disease. The study aims to improve the quality of laboratory diagnosis of LB by direct detection of borreliae in human body fluids mainly in controversial cases (inflammatory cases of unknown ethiology, chronically cases of the Lyme diseases). It can lead to more effective application of the antibiotics, therapeutic algorithm, and improvement of the prognosis of the diseases.

\section{Acknowledgments}

The study was supported by the Slovak Grant Agency VEGA 2/6151/27, 2/0065/09, and by the Slovak Ministry of Health 2007/11SZU-07.

\section{REFERENCES}

1. Demaerschalck I, Ben Messaoud A, De Kesel M, Hoyois B, Lobet Y, Hoet P, et al. Simultaneous presence of different Borrelia burgdorferi genospecies in biological fluids of Lyme disease patients. J Clin Microbiol. 1995 Mar;33(3):602-8.

2. Picken RN, Strle F, Picken MM, Ruzic-Sabljic E, Maraspin V, LotricFurlan S, et al. Identification of three species of Borrelia burgdorferi sensu lato (B. burgdorferi sensu stricto, B. garinii, and B. afzelii) among isolates from acrodermatitis chronica atrophicans lesions. J Invest Dermatol. 1998 Mar;110(3):211-4.

3. Strle F, Ružić-Sabljić E, Cimperman J, Maraspin V, Lotrič-Furlan S. Isolation of Borrelia burgdorferi from normal cerebrospinal fluid in seronegative patients with erythema migrans and peripheral facial palsy. Ann Rheum Dis. 1993 May;52(5):411.

4. Ružić-Sabjić E. Microbiological diagnosis of Lyme borreliosis. Acta Dermatovenerol. 2001;10(4):1-7.

5. Jovičić VL, Grego EM, Lako BL, Ristović BM, Lepšanović ZA, Stajković NT. Improved serodiagnosis of early Lyme borreliosis: immunoblot with local Borrelia afzelii strain. APMIS. 2003 Nov;111(11):1053-9.

6. Schwarzová K, Čižnár I. Spirochetal non-Borrelia-microorganism isolated from Ixodes ricinus. Folia Microbiol. 1996;41(2):175-80.

7. Kmety E, Řeháček J, Výrosteková V. Investigations of ticks for the presence of Borrelia in Czechoslovakia. Zentralbl Bakteriol Mikrobiol Hyg A. 1987 Feb;263(3):468-70.

8. Hovind-Hougen K. Ultrastructure of spirochetes isolated from Ixodes ricinus and Ixodes dammini. Yale J Biol Med. 1984 Jul-Aug;57(4):5438.

9. Postic D, Assous MV, Grimont PA, Baranton G. Diversity of Borrelia burgdorferi sensu lato evidenced by restriction fragment length polymorphism of rrf (5S)-rrl (23S) intergenic spacer amplicons. Int J Syst Bacteriol. 1994 Oct;44(4):743-53.

10. Vancová M, Nebesářová J, Grubhoffer L. Lectin-binding characteristics of a Lyme borreliosis spirochete Borrelia burgdorferi sensu stricto. Folia Microbiol. 2005;50(3):229-38.

11. Epidemiological information system [homepage on the Internet]. EPIS: information pages about communicable diseases occurrence [cited 2009 Sep 14]. Available from: http://www.epis.sk.

12. Mavin S, Milner RM, Evans R, Chatterton JM, Joss AW, Ho-Yen DO. The use of local isolates in Western blots improves serological diagnosis of Lyme disease in Scotland. J Med Microbiol. 2007 Jan;56(Pt 1):47-51.

13. Schwarzová K, Čižnár I. Combined infection of Ixodes ricinus with three Borrelia burgdorferi sensu lato genotypes. Folia Microbiol. 2004;49(3):297-300.

14. Liveris D, Wormser GP, Nowakowski J, Nadelman R, Bittker S, Cooper $\mathrm{D}$, et al. Molecular typing of Borrelia burgdorferi from Lyme disease patients by PCR- restriction fragment length polymorphism analysis. J Clin Microbiol. 1996 May;34(5):1306-9.

Received January 5, 2009 Accepted in revised form April 27, 2009 